\title{
A HEURISTIC ALGORITHM FOR COMPUTING THE POINCARÉ SERIES OF THE INVARIANTS OF BINARY FORMS
}

\author{
DRAGOMIR Ž. ĐOKOVIĆ
}

\begin{abstract}
We propose a heuristic algorithm for fast computation of the Poincaré series $P_{n}(t)$ of the invariants of binary forms of degree $n$, viewed as rational functions. The algorithm is based on certain polynomial identities which remain to be proved rigorously. By using it, we have computed the $P_{n}(t)$ for $n \leq 30$. Dixmier proposed long ago three conjectures concerning these Poincare series. We verify that the first two of them are valid in the above range. As a supplement to this note, we provide a table from which one can easily construct the functions $P_{n}(t)$ for $3 \leq n \leq 30$. Only the portion of the table covering the range $3 \leq n \leq 20$ is actually displayed in the paper.
\end{abstract}

\section{INTRODUCTION}

Let $\mathcal{P}(V)$ be the algebra of polynomial functions on a finite-dimensional complex vector space $V$. Let $\mathcal{P}^{n}(V) \subseteq \mathcal{P}$ be the space of homogeneous polynomials of degree $n$. We shall assume from now on that $V=\mathbf{C}^{2}$ and refer to $\mathcal{P}^{n}\left(\mathbf{C}^{2}\right)$ as the space of binary forms of degree $n$. By fixing a basis, we shall view $\mathcal{P}\left(\mathbf{C}^{2}\right)$ as the polynomial algebra $\mathbf{C}[x, y]$ in the two coordinate functions $x$ and $y$. The natural action of $\mathrm{GL}_{2}(\mathbf{C})$ on $V$ induces an action on $\mathcal{P}\left(\mathbf{C}^{2}\right)$ such that each subspace $\mathcal{P}^{n}\left(\mathbf{C}^{2}\right)$ is stable and the representation of $\mathrm{GL}_{2}(\mathbf{C})$ on it is irreducible.

To simplify the notation, we set $G=\mathrm{SL}_{2}(\mathbf{C})$. One of the main themes of the classical invariant theory was the study of the algebra of $G$-invariants of $\mathcal{P}\left(\mathcal{P}^{n}\left(\mathbf{C}^{2}\right)\right)$, $n=1,2,3, \ldots$ Denote this subalgebra by $\mathcal{I}_{n}=\mathcal{P}\left(\mathcal{P}^{n}\left(\mathbf{C}^{2}\right)\right)^{G}$. This notation is borrowed from the article of R. Howe [5], which gives a modern point of view on this classical subject. The Poincaré series (also known as the Hilbert series) of $\mathcal{I}_{n}$ is the formal power series

$$
P_{n}(t)=\sum_{k \geq 0} \operatorname{dim}\left(\mathcal{I}_{n}^{k}\right) t^{k}
$$

where

$$
\mathcal{I}_{n}^{k}=\mathcal{I}_{n} \cap \mathcal{P}^{k}\left(\mathcal{P}^{n}\left(\mathbf{C}^{2}\right)\right) .
$$

It is well known that this series is in fact the Taylor series of a rational function, which we denote also by $P_{n}(t)$. We consider here the problem of computing efficiently these rational functions.

There are two methods that we find in the recent literature on this subject. First there is a method described in Howe's article and implemented on a computer by P. Saly, Jr. It is based on the classical formulae, due to Cayley and Sylvester, for the coefficients of the above Taylor series and the fact that the concrete form for the denominator of $P_{n}(t)$ is known. Indeed, Dixmier's Conjecture 1 in 3 gives

1991 Mathematics Subject Classification. Primary 13A50, 68W30; Secondary 13P10.

The author was supported in part by the NSERC Grant A-5285. 
simple expressions for the lowest term denominators of the $P_{n}$ 's. (See also Section 6] below.)

The second method is based on an explicit but complicated formula for $P_{n}(t)$ discovered by T. Springer [8. This formula has been used by Brouwer and Cohen [1] and also by Littelmann and Procesi [6].

Our method is completely different and is based on certain conjectural polynomial identities (which are not explicitly given). In spite of this vagueness, the conjectural formulae can be used to write an efficient algorithm to compute the $P_{n}$ 's for small $n$ 's. The algorithm saves time by avoiding the usual tedious procedure of computing many residues one at the time. We used Maple [7 to obtain the formulae for $P_{n}$ for $n \leq 30$. The memory requirements are very modest and the computations can be performed on a PC.

We did not have access to the tables of Brouwer and Cohen (for $n \leq 17$ ) to compare them with our tables. At the end of his article [5], Howe gives two concrete coefficients of $t^{k}$ in the numerator of $P_{n}(t)$, one for $n=19$ and $k=160$ and the other for $n=20$ and $k=84$. The denominators are specified as $\prod_{j=2}^{18}\left(1-t^{2 j}\right)$ for $n=19$, and $\prod_{j=2}^{19}\left(1-t^{j}\right)$ for $n=20$. We find that the first coefficient is correct but the second one should be multiplied by 2 .

In a separate file, we provide the list of the $P_{n}$ 's for $n \leq 30$ as they may be of interest to other researchers. (I wish I had access to such a list when I was doing these computations.) More details about this list are given in Section [6]

\section{THE MAIN ACTORS}

Let $z$ and $t$ be independent commuting variables and $\mathbf{Z}[z, t]$ the corresponding polynomial ring with integer coefficients.

Let $n \geq 3$ be an integer. Set $n=2 s-1$ if $n$ is odd and $n=2 s$ if $n$ is even. The main actors in this paper are the three polynomials $p_{n}, q_{n} \in \mathbf{Z}[z, t]$ and $r_{n} \in \mathbf{Z}[t]$. The first two are defined, for odd $n$, by

$$
p_{n}(z, t)=\prod_{i=1}^{s}\left(1-t z^{2 i-1}\right), \quad q_{n}(z, t)=\prod_{i=1}^{s}\left(z^{2 i-1}-t\right),
$$

and, for even $n$, by

$$
p_{n}(z, t)=\prod_{i=1}^{s}\left(1-t z^{2 i}\right), \quad q_{n}(z, t)=\prod_{i=1}^{s}\left(z^{2 i}-t\right) .
$$

In both cases, $s$ is the $t$-degree of $p_{n}$ (and $q_{n}$ ), i.e., its degree as a polynomial in the variable $t$. Denote by $m$ the $z$-degree of $p_{n}$ (and $q_{n}$ ). Thus, $m=s^{2}$ if $n$ is odd and $m=s(s+1)$ if $n$ is even.

Observe that

$$
z^{m} p_{n}\left(z^{-1}, t\right)=q_{n}(z, t), \quad(-t)^{s} p_{n}\left(z, t^{-1}\right)=q_{n}(z, t),
$$

and, consequently,

$$
z^{m}(-t)^{s} p_{n}\left(z^{-1}, t^{-1}\right)=p_{n}(z, t) .
$$

These formulae remain valid when the letters $p$ and $q$ are interchanged.

The third polynomial is defined by

$$
r_{n}(t)=\prod_{i=2}^{n-1}\left(1-t^{2 i}\right)
$$


if $n$ is odd, and by

$$
r_{n}(t)=(1+t) \prod_{i=2}^{n-1}\left(1-t^{i}\right)
$$

if $n$ is even.

Finally, set

$$
\varphi_{n}(z, t)=z^{m-2}\left(z^{2}-1\right) r_{n}(t) .
$$

It is easy to verify that

$$
z^{2 m-2} \varphi_{n}\left(z^{-1}, t\right)=-\varphi_{n}(z, t), \quad t^{d} \varphi_{n}\left(z, t^{-1}\right)=(-1)^{n} \varphi_{n}(z, t),
$$

and, consequently,

$$
z^{2 m-2} t^{d} \varphi_{n}\left(z^{-1}, t^{-1}\right)=(-1)^{n+1} \varphi_{n}(z, t),
$$

where $d$ is the degree of $r_{n}(t)$. Thus $d=2 s(n-2)$ for $n$ odd and $d=s(n-1)$ for $n$ even.

\section{BASIC CONJECTURE}

In this section we state our basic working conjecture, which will be taken for granted in the remaining part of the paper. It is supported by extensive computer calculations.

Let $I_{n}=<p_{n}, q_{n}>$ be the ideal of $\mathbf{Z}[z, t]$ generated by $p_{n}$ and $q_{n}$. We conjecture that $\varphi_{n} \in I_{n}$. More precisely,

Conjecture 3.1. There exist unique polynomials $a_{n}, b_{n} \in \mathbf{Z}[z, t]$ of $z$-degree $m-2$ such that

$$
\varphi_{n}(z, t)=a_{n}(z, t) q_{n}(z, t)+b_{n}(z, t) p_{n}(z, t) .
$$

(The uniqueness is clear because $p_{n}$ and $q_{n}$ are relatively prime.)

Let us give an example. If $n=5$ then $s=3, m=9$ and $\varphi=a q+b p$, where

$$
\begin{aligned}
\varphi= & z^{7}\left(z^{2}-1\right)\left(1-t^{4}\right)\left(1-t^{6}\right)\left(1-t^{8}\right), \\
p= & (1-t z)\left(1-t z^{3}\right)\left(1-t z^{5}\right), \\
q= & (z-t)\left(z^{3}-t\right)\left(z^{5}-t\right), \\
a= & \left(1-t^{6}+t^{12}\right)-t\left(1+t^{2}-t^{6}\right)\left(1-t^{2}-t^{6}\right) z+t^{2}\left(1-t^{6}+t^{8}\right) z^{2} \\
& -t\left(1-t^{4}\right)\left(1-t^{6}-t^{8}\right) z^{3}+t^{2}\left(1-t^{4}\right)\left(1+t^{2}-t^{8}\right) z^{4}-t^{5}\left(1-t^{2}+t^{8}\right) z^{5} \\
& +t^{2}\left(1+t^{4}-t^{6}\right)\left(1-t^{4}-t^{6}\right) z^{6}-t^{3}\left(1-t^{6}+t^{12}\right) z^{7}, \\
b= & t^{3}\left(1-t^{6}+t^{12}\right)-t^{2}\left(1+t^{4}-t^{6}\right)\left(1-t^{4}-t^{6}\right) z+t^{5}\left(1-t^{2}+t^{8}\right) z^{2} \\
& -t^{2}\left(1-t^{4}\right)\left(1+t^{2}-t^{8}\right) z^{3}+t\left(1-t^{4}\right)\left(1-t^{6}-t^{8}\right) z^{4}-t^{2}\left(1-t^{6}+t^{8}\right) z^{5} \\
& +t\left(1+t^{2}-t^{6}\right)\left(1-t^{2}-t^{6}\right) z^{6}-\left(1-t^{6}+t^{12}\right) z^{7} .
\end{aligned}
$$

By applying the substitution $z \rightarrow z^{-1}$ to (3.1) and by using the identities (2.1) and (2.2), we obtain that

$$
b_{n}(z, t)=-z^{m-2} a_{n}\left(z^{-1}, t\right) .
$$

Similarly, the substitution $t \rightarrow t^{-1}$ gives

$$
b_{n}(z, t)=(-1)^{n+s} t^{d-s} a_{n}\left(z, t^{-1}\right) .
$$

From the last two equations we deduce that

$$
z^{m-2} t^{d-s} a_{n}\left(z^{-1}, t^{-1}\right)=(-1)^{n+s+1} a_{n}(z, t) .
$$


We can write

$$
a_{n}(z, t)=\sum_{i=0}^{m-2} \alpha_{k}(t) z^{k}, \quad b_{n}(z, t)=\sum_{i=0}^{m-2} \beta_{k}(t) z^{k},
$$

where $\alpha_{k}, \beta_{k} \in \mathbf{Z}[t]$. Then the equation (3.2) gives

$$
\beta_{k}(t)=-\alpha_{m-2-k}(t), \quad 0 \leq k \leq m-2
$$

and (3.4) gives

$$
\alpha_{m-2-k}(t)=(-1)^{n+s+1} t^{d-s} \alpha_{k}\left(t^{-1}\right), \quad 0 \leq k \leq m-2 .
$$

Hence, the equation (3.1) can now be rewritten as

$$
\varphi_{n}(z, t)=\sum_{k=0}^{m-2} \alpha_{k}(t)\left(z^{k} q_{n}(z, t)-z^{m-2-k} p_{n}(z, t)\right) .
$$

By setting $z=0$ in (3.8), we obtain that $\alpha_{m-2}(t)=(-t)^{s} \alpha_{0}(t)$. By combining this with the equation (3.7) for $k=0$, we obtain that

$$
\alpha_{0}(t)=(-1)^{n+1} t^{d-2 s} \alpha_{0}\left(t^{-1}\right) .
$$

Apparently the computation of the polynomials $a_{n}(z, t)$ is a difficult job. Fortunately, we do not need to know these polynomials explicitly but only the polynomials $a_{n}(0, t)=\alpha_{0}(t)$. The main point of our algorithm is that the polynomials $\alpha_{0}(t)$ can be computed efficiently.

For the curious reader, let us throw in one more intriguing conjecture (which plays no role whatsoever in this paper).

Conjecture 3.2. $I_{n} \cap \mathbf{Z}[t]$ is the principal ideal of $\mathbf{Z}[t]$ generated by the polynomial

$$
\left(1-t^{2}\right) \prod_{i=1}^{n-1}\left(1-t^{2 i}\right), \quad(1+t) \prod_{i=1}^{n-1}\left(1-t^{i}\right), \quad \prod_{i=1}^{n-1}\left(1-t^{i}\right),
$$

according to whether $n$ is odd, congruent to 2 modulo 4, or divisible by 4.

This conjecture makes sense (and is true) also when $n$ is equal to 1 or 2 .

\section{The INTEGRAL FORMUla}

The Molien-Weyl integral formula for $P_{n}(t)$ can be written in the following form (see [2])

$$
P_{n}(t)=\frac{1}{2 \pi i} \int_{|z|=1} f_{n}(z, t) \frac{\mathrm{d} z}{z},
$$

where

$$
f_{n}(z, t)=\frac{1-z^{-2}}{\prod_{k=0}^{n}\left(1-t z^{n-2 k}\right)} .
$$

The integration is to be performed over the unit circle in the counterclockwise direction and it is assumed that $|t|<1$.

Let us define yet another function

$$
g_{n}(z, t)=\frac{z^{m-2}\left(z^{2}-1\right)}{p_{n}(z, t) q_{n}(z, t)} .
$$


For odd $n, f_{n}(z, t)=g_{n}(z, t)$ and, for even $n, f_{n}(z, t)=g_{n}(z, t)(1-t)^{-1}$. Since the integration variable is $z$, the factor $(1-t)^{-1}$ can be inserted at the very end of the computation. Thus it suffices to compute the integral

$$
Q_{n}(t)=\frac{1}{2 \pi i} \int_{|z|=1} g_{n}(z, t) \frac{\mathrm{d} z}{z} .
$$

The formula (3.1) gives

$$
r_{n}(t) g_{n}(z, t)=\frac{a_{n}(z, t)}{p_{n}(z, t)}+\frac{b_{n}(z, t)}{q_{n}(z, t)} .
$$

Hence,

$$
r_{n}(t) Q_{n}(t)=\frac{1}{2 \pi i} \int_{|z|=1} \frac{a_{n}(z, t)}{p_{n}(z, t)} \frac{\mathrm{d} z}{z}+\frac{1}{2 \pi i} \int_{|z|=1} \frac{b_{n}(z, t)}{q_{n}(z, t)} \frac{\mathrm{d} z}{z} .
$$

The second integral is 0 since all the poles are inside the unit circle (and the residue at $\infty$ is 0 ). The integrand of the first integral has all of its poles outside the unit circle except for the simple pole at $z=0$. Since $a_{n}(0, t)=\alpha_{0}(t)$ and $p_{n}(0, t)=1$, we obtain that

$$
Q_{n}(t)=\frac{\alpha_{0}(t)}{r_{n}(t)}
$$

Hence, the computation of $P_{n}(t)$ is reduced to computing the polynomial $\alpha_{0}(t)$. Since $Q_{n}(0)=r_{n}(0)=1$, we deduce that $\alpha_{0}(0)=1$. The formula (3.9) now implies that $\alpha_{0}$ has degree $d-2 s$. This is in agreement with the well known fact (it was known to Sylvester 9]) that the rational function $P_{n}(t)$ has degree $-n-1$.

If $n$ is odd, then $\alpha_{0}(t)$ is a palindromic polynomial, i.e., its coefficients are symmetric about the midpoint. If $n$ is even, then $\alpha_{0}(1)=0$, i.e., $\alpha_{0}(t)$ is divisible by $1-t$.

Let us emphasize that the main feature of our algorithm described above is that we compute the above integral at one fell swoop, avoiding the tedious procedure of computing all the residues for the poles inside the unit circle one at the time.

\section{How to COMPute $\alpha_{0}$ ?}

Let me start by quoting the Rule \#1 from the list of useful tips on Jean-Charles Faugère's home page: "In a first time try to compute modulo $p$ where $p$ is a small prime." I learned this rule on my own (i.e., the hard way).

To compute $\alpha_{0}(t)$ we used the Maple package called "LinearAlgebra" and its subpackage called "Modular". First we choose a big prime, $l$, in the data type integer[4]. The largest one is $l=65521$. We perform computations modulo this prime. We generate random integer mod $l$ inputs for the variable $z$ and plug it into our equation (3.8). We need only $m-1$ inputs since there are $m-1$ unknown $\alpha_{k}$ 's.

Thus we obtain a system of $m-1$ equations which are linear in the $\alpha_{k}$ 's. But these are still polynomial equations as they contain the variable $t$. We chose a random value, say $\tau$ modulo $l$, for the variable $t$ and plug it into this system of equations. Now we have just a small system of linear equations to solve for the unknowns $\alpha_{k}(\tau)$. This job can be easily handled by "Modular". We are only interested in the value $\alpha_{0}(\tau)$.

Next recall that we know the degree of $\alpha_{0}$; it is equal to $d-2 s$. Thus we have to repeat the above calculation $d-2 s+1$ times to get the values $\alpha_{0}(\tau)$ for $d-2 s+1$ different inputs $\tau$. Having done this, the $d-2 s+1$ unknown coefficients of $\alpha_{0}(t)$ can 
be easily computed by solving the corresponding Van der Monde system of linear equations. Of course, this means that at this stage we know these coefficients modulo our prime $l$.

Next we have to repeat the whole calculation above with several distinct primes. We used the largest seven primes available:

$$
65521,65519,65497,65479,65449,65447,65437 .
$$

This is necessary when $n$ is near the high end of our range because some of the coefficients of $\alpha_{0}(t)$ for $n=29$ have 30 digits. The remaining task is to lift these modular solutions to a genuine solution over Z Z For this purpose, "Modular" provides the "ChineseRemainder" command which makes the task very easy.

In the hardest case, $n=29$, the computation of $\alpha_{0}(t)$ modulo the above seven primes (performed one after the other) took almost 5 hours on the SunBlade workstation running a single R10000 CPU at $250 \mathrm{MHz}$ with 8 GB of RAM.

Certainly, if Conjecture 3.1 was false these computations, which involve so many random choices, would not produce a meaningful result. Moreover, the polynomials $\alpha_{0}(t)$ have certain symmetry properties. As mentioned earlier, if $n$ is odd then $\alpha_{0}(t)$ is a palindromic polynomial. If $n$ is even, then $\alpha_{0}(t)$ is divisible by $1-t$, according to one of the Dixmier's conjectures. Finally, if $n$ is divisible by 4 , then $\alpha_{0}(t)$ is also divisible by $1+t^{s-1}$, again by Dixmier. This is all true in the cases that we have computed.

If $n \geq 3$ then $t=1$ is a pole of $P_{n}(t)$ of order $n-2$ and the limit of $(1-t)^{n-2} P_{n}(t)$ as $t \rightarrow 1$ has been computed by Hilbert 4. We have verified that our formulae for $P_{n}(t)$ agree with his result.

\section{Description of the Coefficient table}

There exist unique relatively prime polynomials $A_{n}, B_{n} \in \mathbf{Z}[t]$, with $A_{n}(0)=$ $B_{n}(0)=1$, such that

$$
P_{n}(t)=\frac{A_{n}(t)}{B_{n}(t)} .
$$

In this notation, Dixmier's Conjecture 1 asserts that $B_{n}(t)=r_{n}(t) /\left(1+t^{s-1}\right)$ if $n$ is divisible by 4 , and $B_{n}(t)=r_{n}(t)$ otherwise. He verified this conjecture for $n \leq 16$ and our computations show that it is valid for $n \leq 30$.

We propose the following additional conjecture:

Conjecture 6.1. The polynomials $A_{n}(t)$ are irreducible for $n \geq 5$.

Since the denominators $B_{n}(t)$ are easy to write down, we provide only the information necessary to construct the numerators $A_{n}(t)$ for $3 \leq n \leq 30$.

If $n$ is odd, then $A_{n}(t)=\alpha_{0}(t)$. Now assume that $n$ is even. Then $\alpha_{0}(t)=$ $(1-t) \bar{\alpha}_{0}(t)$ for some $\bar{\alpha}_{0} \in \mathbf{Z}[t]$ and $P_{n}(t)=\bar{\alpha}_{0}(t) / r_{n}(t)$. Thus $A_{n}(t)=\bar{\alpha}_{0}(t)$ if $n \equiv 2(\bmod 4)$. Finally, if $n \equiv 0(\bmod 4)$, then $\bar{\alpha}_{0}(t)$ is divisible by $1+t^{s-1}$, and we have $A_{n}(t)=\bar{\alpha}_{0}(t) /\left(1+t^{s-1}\right)$.

The degree of $A_{n}$ is always even and we shall denote it by $2 \delta$. We have $\delta=$ $2 s(s-2)$ for $n$ odd, $2 \delta=s(2 s-3)-1$ for $n \equiv 2(\bmod 4)$, and $\delta=s(s-2)$ for $n \equiv 0(\bmod 4)$. These numerators are palindromic polynomials

$$
A_{n}(t)=c_{0}+c_{1} t+\cdots+c_{\delta-1} t^{\delta-1}+c_{\delta} t^{\delta}+c_{\delta-1} t^{\delta+1}+\cdots+c_{1} t^{2 \delta-1}+c_{0} t^{2 \delta}
$$

and so it suffices to store roughly half of their coefficients. 
For each $n$ in the range $3 \leq n \leq 30$ we record the coefficients $c_{0}=1, c_{1}, \ldots, c_{\delta}$ of $A_{n}$ in that order. They are stored as a Maple table $A[3 . .30]$.

For example, if $n=5$ then $s=3, \delta=6, A_{5}=1-t^{6}+t^{12}$. This is recorded in our table as

$$
A[5]=[1,0,0,0,0,0,-1] .
$$

Assume that $n \geq 3$ is odd. Then it is known that $c_{i}=0$ for odd $i$ 's as well as for $i=2$. Dixmier $[3]$ shows that $c_{4}$ and $c_{8}$ are nonnegative, and so are $c_{6}$ for $n \geq 15$ and $c_{10}$ for $n \geq 9$. We can prove that in fact

$$
c_{4}=\left[\frac{n-3}{6}\right] \text {. }
$$

It may be useful to obtain similar formulae for $c_{6}, c_{8}$, etc.

\section{Appendix: Table $A[3 . .20]$}

We display here the coefficients $c_{0}=1, c_{1}, \ldots, c_{\delta}$ of the numerator $A_{n}(t)$. Recall that the degree of $A_{n}(t)$ is $2 \delta$. The display includes only the values $n=3,4, \ldots, 20$. The full table with the range $n=3,4, \ldots, 30$ is available from the author on request. 


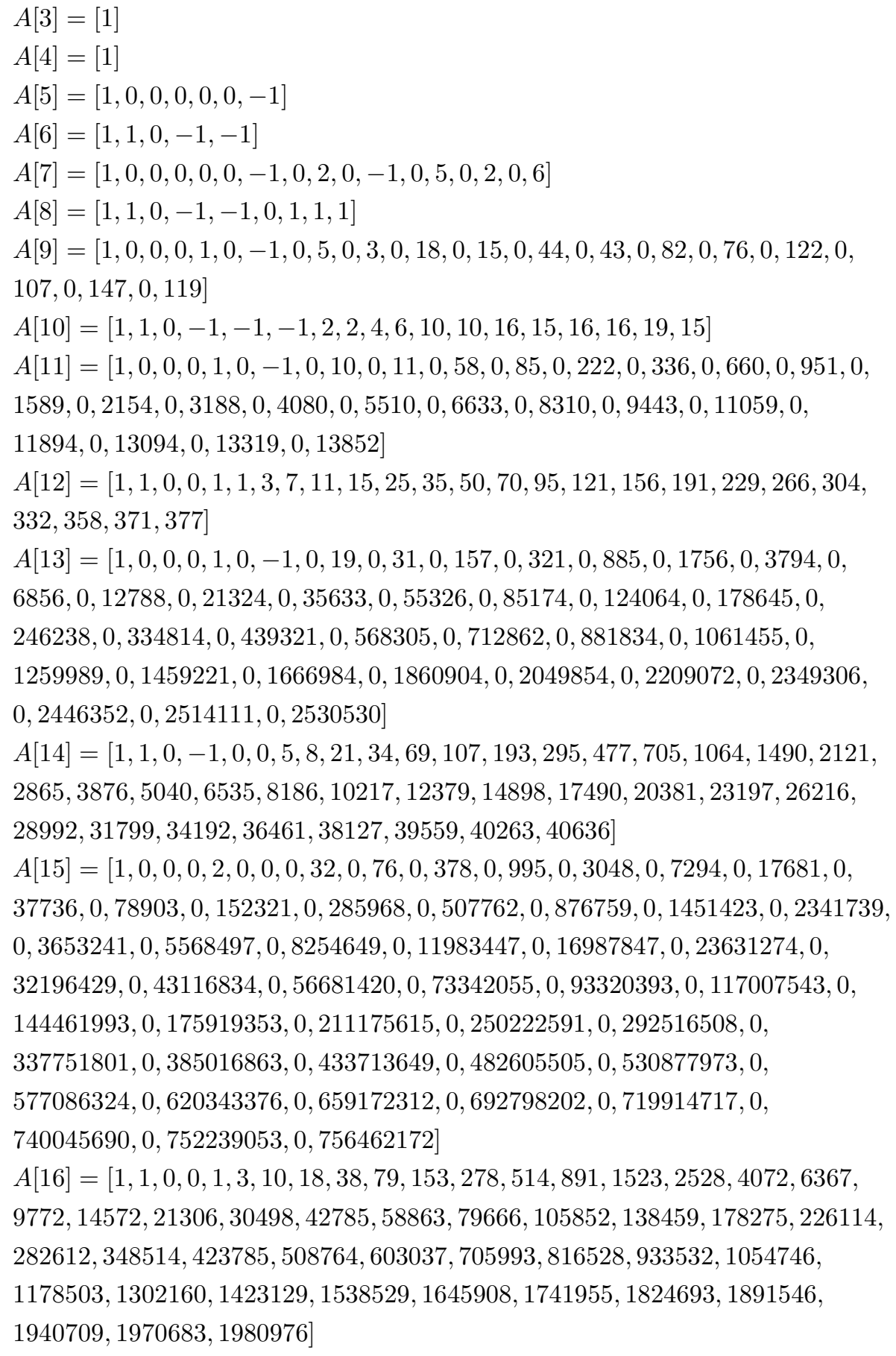


$A[17]=[1,0,0,0,2,0,0,0,50,0,156,0,844,0,2716,0,9280,0,26055,0$, $70846,0,173224,0,405183,0,883551,0,1847356,0,3669433,0,7024773,0$, 12919848, 0, 23019526, 0, 39697193, 0, 66608244, 0, 108748704, 0, 173371011, $0,270001994,0,411791616,0,615371715,0,902700319,0,1300556398,0$, $1842885348,0,2569619659,0,3529481145,0,4777707107,0,6379225544,0$, 8404807944, 0, 10934524315, 0, 14051849433, 0, 17847385164, 0, 22410522390, $0,27833575972,0,34200807232,0,41593316021,0,50075498973,0$, $59701312756,0,70498510402,0,82477120228,0,95612069584,0$, $109854817368,0,125115124389,0,141276752124,0,158179073390,0$, $175637520454,0,193425539961,0,211299997259,0,228983624510,0$, 246195050087, 0, 262631400199, 0, 278002047047, 0, 292010632283, 0, $304391087240,0,314888097678,0,323293686822,0,329425050810,0$, $333160013567,0,334411422423]$

$A[18]=[1,1,0,-1,1,2,11,20,60,122,292,573,1199,2264,4307,7692,13639$, 23121, 38688, 62619, 99808, 154969, 236962, 354532, 522744, 756614, 1080091, 1517149, 2103843, 2875718, 3883971, 5178127, 6826649, 8894132, 11466985, 14623600, 18466440, 23083747, 28588450, 35071007, 42645944, 51394256, 61419462, 72778864, 85549508, 99748823, 115411657, 132499497, 150990759, 170778600, 191774520, 213796608, 236686776, 260188811, 284080692, $308043009,331804545,355002792,377341521,398442572,418014893$, 435697784, 451237581, 464324429, 474773647, 482354991, 486977592, $488512665]$ 
$A[19]=[1,0,0,0,2,0,1,0,76,0,296,0,1763,0,6738,0,25712,0,82893,0$, $252012,0,694765,0,1807368,0,4392969,0,10154779,0,22296093,0$, 46930799, 0, 94802787, 0, 184822672, 0, 348299741, 0, 636837951, 0, 1131559371, 0, 1959027689, 0, 3309329549, 0, 5465457626, 0, 8835569146, 0, 14002953513, 0, 21778902561, 0, 33281420196, 0, 50015102190, 0, 73986433683, 0, 107815040622, $0,154890725357,0,219515780752,0,307103168571,0,424341546490,0$, $579425240340,0,782222455286,0,1044516156680,0,1380143077777,0$, $1805211986577,0,2338168202449,0,2999945060393,0,3813898895206,0$, 4805843429850, 0, 6003797685274, 0, 7437855144286, 0, 9139694108669, 0, $11142247265979,0,13478942963820,0,16183166152183,0,19287230767266$, 0, 22821658964018, 0, 26813925195712, 0, 31287624164380, 0, 36261071290807, $0,41746472475910,0,47748507080547,0,542636466777477,0,61278874674037$, $0,68771319597259,0,76707272862777,0,85042295486194,0,93720678352722$, $0,102676146969267,0,111831862933442,0,121101792448393,0$, 130391278393710, 0, 139599058259333, 0, 148618354053166, 0 , $157339436459345,0,165651094224795,0,173443559767368,0$, 180610146679903, 0, 187050288671933, 0, 192671089114197, 0, $197390193869982,0,201136986494180,0,203855025614692$, 0, 205502649265697, 0, 206054755643582]

$A[20]=[1,1,0,0,2,5,17,40,100,232,544,1199,2599,5365,10770,20867$, $39312,71826,128004,222286,377375,626606,1019690,1627231,2550571$, 3929105, 5955664, 8888409, 13072800, 18958777, 27131530, 38333433, 53503793, 73805811, 100673389, 135839979, 181391167, 239789025, 313924970, 407134237, $523239787,666546890,841871660,1054502755,1310204347,1615137665$, 1975827494, 2399034646, 2891684840, 3460685330, 4112816074, 4854497808, $5691649536,6629418196,7672023583,8822464074,10082371655,11451725459$, $12928740940,14509620528,16188514360,17957332700,19805806095$, 21721392176, 23689449970, 25693256426, 27714311797, 29732461463, $31726325603,33673521588,35551188212,37336279422,39006154571$, 40538894181, 41913908026, 43112232559, 44117095575, 44914144829, $45491928155,45842000355,45959277535]$

\section{REFERENCES}

[1] A.E. Brouwer and A.M. Cohen, The Poincaré series of the polynomial invariants under $\mathrm{SU}_{2}$ in its irreducible representation of degree $\leq 17$, preprint of the Mathematisch Centrum, Amsterdam, 1979.

[2] H. Derksen and G. Kemper, Computational Invariant Theory, Springer-Verlag, New York, 2002. 
[3] J. Dixmier, Quelques résultats et conjectures concernant les séries de Poincaré des invariants des formes binaires, in "Lecture Notes in Mathematics," Vol. 1146, pp. 127-160, SpringerVerlag, Berlin, 1985.

[4] D. Hilbert, Über die vollen Invariantensysteme, Math. Ann. 36 (1890), 473-534.

[5] R. Howe, The Classical Groups and Invariants of Binary Forms, in The Mathematical Heritage of Hermann Weyl, Vol. 48, Proc. Symp. Pure Math., Amer. Math. Soc., Providence, R.I., 1987.

[6] P. Littelmann and C. Procesi, On the Poincaré series of the invariants of binary forms, J. Algebra 133 (1990), 490-499.

[7] MAPLE, Maplesoft, Waterloo, Ontario.

[8] T.A. Springer, On the invariant theory of $\mathrm{SU}_{2}$, Indag. Math. 42 (1980), 339-345.

[9] J.J. Sylvester and F. Franklin, Tables of the generating functions and groundforms for the binary quantics of the first ten orders, Amer. J. Math. 2 (1879), 223-251.

Department of Pure Mathematics, University of Waterloo, Waterloo, Ontario, N2L 3G1, CANADA

E-mail address: djokovic@uwaterloo.ca 